\title{
Optimality Modeling and Explanatory Generality
}

\author{
Angela Potochnik $\$$
}

The optimality approach to modeling natural selection has been criticized by many biologists and philosophers of biology. For instance, Lewontin (1979) argues that the optimality approach is a shortcut that will be replaced by models incorporating genetic information, if and when such models become available. In contrast, I think that optimality models have a permanent role in evolutionary study. I base my argument for this claim on what I think it takes to best explain an event. In certain contexts, optimality and game-theoretic models best explain some central types of evolutionary phenomena.

1. Introduction. The optimality approach offers a way to model natural selection purely phenotypically, without directly representing the system of genetic transmission. This approach includes both optimality models and game-theoretic models, which are used when trait fitnesses are frequency-dependent. One determines the range of possible values for some phenotype and the fitness function relating these phenotypes to the environment. Based on this information, the model predicts which phenotypic value(s) will predominate in the population, given enough time in that environment. Modeling long-term evolutionary change in this manner results in information regarding the effect of the selection pressure(s) at work and any constraints arising from, for example, the process of genetic transmission or development.

Various concerns about the reliability and long-term value of optimality modeling have been raised by population biologists and philosophers of biology. One thought is that, though optimality models are a helpful substitution when genetic information is unavailable, they should eventually be replaced by models that explicitly incorporate genetic infor-

$\dagger$ To contact the author, please write to: Department of Philosophy, Stanford University, Stanford, CA 94305-2155; e-mail: potochnik@stanford.edu.

$\$$ Thanks to Michael Friedman, Helen Longino, Michael Weisberg, and especially Elliott Sober for comments on earlier drafts of this paper.

Philosophy of Science, 74 (December 2007) pp. 680-691. 0031-8248/2007/7405-0011 \$10.00

Copyright 2007 by the Philosophy of Science Association. All rights reserved. 
mation. Lewontin (1979) articulates such a view. He takes optimality modeling to be a shortcut approach that does not truly capture the dynamics of evolutionary change. In his view, a truly dynamical theory of evolution will "predict the evolutionary trajectory of the community . . . on a purely mechanical basis" $(1979,6)$. This requires the incorporation of genetic dynamics into models of natural selection. Lewontin thus thinks that, if and when it is possible, the optimality approach should be replaced by models that explicitly represent the dynamics of genetic transmission.

In contrast to this position, I here develop a case for employing optimality models on a long-term basis, regardless of the availability of genetic information. It is true that phenotypic models shortcut the genetic dynamics crucial to evolutionary change. However, optimality models capture other important aspects of the dynamics of evolutionary change. In particular, these models represent the fitness-conferring interactions between organisms and their environment. Because of this, and because of features of scientific explanation for which I will argue below, I think that in some important contexts, optimality models best explain certain evolutionary phenomena. ${ }^{1}$ In my view, the long-term importance of the optimality approach is assured by its role in evolutionary explanation.

2. Generality, Complexity, and Explanation. Advocates of the causal account of explanation argue that causal factors leading to an event comprise the explanation of that event. From this, the question arises: which of the causal factors leading to an event should be included in an explanation of that event? Simply claiming that explanation is causal does nothing to sort out which parts of a welter of causal detail should be included in an explanation. Even if all causal factors are potentially of explanatory relevance, there is the issue of which of these factors should, ideally, be included in an explanation of the event. Here I sketch an answer this question.

One possibility is that an explanation should be maximally inclusive of causal factors leading to the event to be explained. The idea behind this is that a causal explanation stands only to benefit from the inclusion of

1. The issue at stake is not whether optimality and game-theoretic models yield accurate predictions. I instead ask whether (sufficiently accurate) phenotypic models offer explanations that, in some cases, are better than other types of explanations, including those offered by (sufficiently accurate) models incorporating genetic information. See Sober (2003) on the distinction between the questions of truth and explanatory worth. Relatedly, my argument is not one of abductive inference, or inference to the best explanation. I presume throughout this paper that the candidate explanations are all taken to be true. The question is not which model we should believe is accurate of the phenomenon to be explained, but which (accurate) model best explains the phenomenon. 
additional information about the causes of the event to be explained. Simply string all available causal information together, and the result is the best explanation that is currently possible. Yet I do not think that the strategy of maximal inclusion leads to the best explanations. This is most clear when explaining events that result from highly complex causal processes.

Many events result from the combination and interaction of a large number of causal factors. For such events, there are many factors that influence the event to be explained. Cumulative evolutionary change is exemplary of such a complex causal process. Consider, for instance, the fixation of some physical trait in a population, due to evolution by natural selection. For such a case, causal factors include aspects of the population's genotypes, of its environment, and of the developmental processes leading from genotype to phenotype. Additional factors include population size, amount of inbreeding and migration; the list goes on. The strategy of maximal inclusion is not effective for such complex causal processes, for it would result in exceedingly involved explanations. Even when constructing such an elaborate explanation is even possible, we seldom or ever explain this way. Instead, some subset of the multitude of causal factors is focused upon, circumscribed temporally and often in other ways as well.

If the principle of maximal inclusion is wrong, then explanations should instead feature some subset of the causal factors leading to the event to be explained. This is so at least for events that result from a complex causal process, such as evolution. In my view, this is a natural result. No explanation can trace back the causal chain indefinitely; maximal inclusion is in this sense unobtainable. Aiming to include only some subset of the causal factors places a limit on the cognitive load produced by ideal explanations. This accords well with actual scientific practice. It respects the division of labor that exists among distinct fields and subfields of science. A population with a particular physical trait-my example heremay be studied variously by developmental theorists, evolutionary ecologists, and population geneticists. Each focuses upon a different subset of the causes that influence the outcome.

If I am correct that the best explanation of an event is comprised of a subset of causal factors, then the question arises: What rules determine which causal factors should be included in the explanation and which should be neglected? ${ }^{2}$ In my view, the best explanation of an event $E$ is a model that

2. Strevens (2004) answers this question by developing a restrictive notion of difference making that eliminates from an explanation much of the causal network leading to the event to be explained. However, I do not think Strevens's account sufficiently 
1. represents the causes of $E$ that figure into the causal relationship of interest in the particular context of inquiry at hand;

2. satisfies the criteria of explanatory adequacy; and

3. is maximally general within these constraints.

The first and second conditions determine what information should be included in the best explanation of an event. The third condition decides what information should be neglected by an explanation. ${ }^{3}$

I have argued that maximal inclusion of causal factors does not result in the best explanations. The alternative of citing only a subset of causes creates a tricky situation, though. This results in the systematic neglect of some causes of an event from that event's explanation. The reason that such neglect is not problematic, in my view, is that which causes should be included in an explanation varies, even for a single explanandum. The context of inquiry is determined by the interests of those seeking the explanation. Different research programs approach an explanatory problem from different angles. These interests help to determine which causes must be included in the best explanation of the event in question. Those parts of the causal process picked out by the applicable research interests are the causal factors that an explanation must include, in that context of inquiry.

As an example, consider explaining why most or all members of a population have some particular phenotype. Approaching the question with a research interest in development and with an interest in evolution result in two different explanatory strategies, familiar from discussions of evolutionary explanation. These regard the proximate and ultimate (or distal) causes of the trait, respectively (Mayr 1961). In my view, these distinct explanatory strategies, each focusing on a different subset of causal factors, exist because the explanations are formulated in two different contexts of inquiry. A developmental biologist is primarily interested in the causal relationships involved in development. A population biologist, in contrast, is primarily interested in the causes and effects of selection and transmission. Such distinct research foci result in distinct explanatory strategies.

The role of the context of inquiry in determining what causes should be included in an explanation yields the first condition that must be met

restricts the factors cited in an explanation. Maximal inclusion of just Strevens's difference makers faces the same problems discussed above.

3. This formulation assumes that explanations are provided by models. Such a formulation is adequate for my present purposes, for I aim to establish the explanatory importance of a type of population biology model. However, I am not committed to the idea that only models explain events. 
by a fully satisfactory, 'best' explanation. This is the requirement that an explanation represent the causes of the event to be explained that figure into the causal relationship of particular interest in the context of inquiry at hand. The second condition limits the degree to which the context of inquiry can shape the explanation. The best explanation of an event must meet what I term the criteria of explanatory adequacy.

Imagine that research interests steer one toward a set of causes that would not have led to the event to be explained, had they not occurred in conjunction with certain other factors. The best explanation of an event must, it seems, cite some set of factors that actually does account for the occurrence of the event. The failure of a candidate explanation to do so suggests that it cannot be the best explanation of that event. For instance, an evolutionary ecologist may focus upon the influence of natural selection on some physical trait in a population. Yet, if selection would have resulted in the members of the population having trait $P_{1}$, but because of a lack of genetic variability they instead have trait $P_{2}$, then information about the selection pressure cannot by itself fully explain the population having trait $P_{2}$.

Such concerns lead to my criteria of explanatory adequacy. These set the standard of what can qualify as a fully adequate explanation, regardless of the context of inquiry. The two criteria are:

1. $\operatorname{Pr}\left(E \mid C_{\text {expl }}\right) \approx \operatorname{Pr}(E \mid C)$

2. $\operatorname{Pr}\left(E \mid C_{\text {expl }} \approx \operatorname{Pr}\left(E \mid\left(C_{\text {expl }} \wedge C_{k}\right)\right.\right.$ for all $C_{k}$.

$C_{\text {expl }}$ is the set of causes cited in the candidate explanation of event $E$. $C$ is the broader set of all causal factors that influence $E$. $C_{k}$ represents each event that is a causal influence on $E$. For this second criterion, these $C_{k}$ should be considered both singularly and in combination. These two criteria amount to the idea that, to be fully adequate, an explanation of an event $E$ must more or less screen off $E$ from all additional causal factors. For complex causal processes, there potentially will be many subsets of causal factors that satisfy these criteria, thereby meeting the second condition for a best explanation.

I take these criteria of explanatory adequacy to be rather intuitive. A fully satisfactory explanation should not omit any causal information that, if included, would drastically change the expected probability of the event to be explained. There is the sense that an explanation is not entirely satisfactory if it wholly neglects a causal factor that makes the event to be explained much more probable - or, for that matter, much less probable. For this reason, for example, a developmental explanation for the commonality of a phenotypic trait in the population of interest must, besides facts of development, give at least a minimal amount of genetic information. The explanation must at least cite the genetic composition 
of the population. Without information about the proportion of the population with the genotype(s) that cause the organism to develop the phenotypic trait in question, the frequency of the trait cannot be predicted from developmental information.

The third and final condition to be satisfied by best explanations regards not what should be included in an explanation, but what should be neglected. The best explanation of an event is the most general explanation that satisfies the first two conditions. The notion of generality that I have in mind here is similar to Strevens's, according to which the generality of a model is "proportional to the standard measure (in the mathematical sense) of the set of possible physical systems satisfying the model" (2004, 26). I prefer this formulation in terms of the possible - rather than actual-systems satisfying a model, for this correlates the generality of a model with the amount of causal detail it incorporates (Strevens 2004).

The causal factors that should be included in an explanation are determined by what sort of causal relationship is of particular interest and by the criteria of explanatory adequacy. It is best for an explanation to avoid citing information beyond what these considerations require. Avoiding all information extraneous to the causal relationship of interest and not required for explanatory adequacy makes as perspicuous as possible the causal relationship of interest in the context at hand. It is this relationship that best explains, in the given context of inquiry. Maximizing generality in this way results in an explanation that is applicable to the largest set of possible systems that manifest the causal relationship of interest.

Arguments for the explanatory worth of generality are familiar from advocates of high level explanation (Putnam 1975; Garfinkel 1981), as well as from Strevens's (2004) causal account of explanation. Arguments against the idea that generality is advantageous to an explanation have focused on the idea that explanations sometimes benefit from greater specificity and a corresponding loss of generality (Jackson and Pettit 1992; Sober 1999). Yet the role of generality in the present account is not susceptible to this criticism. This is due to the central role of context in determining the preferable explanation. My claim is not that a certain, most general explanation always best explains an event. Instead, the degree and type of an explanation's allowable generality varies, depending upon the causal relationship that is of interest in the particular context of inquiry at hand.

The conditions I have outlined favor explanations that focus only on what I will call a modular part of the causal process that led to the event to be explained. I have argued that fully satisfactory explanations should give information about a causal relationship that is determined by the interests of the explanation seekers. Such causal relationships potentially 
recur in multiple systems that may differ from one another in other regards. The causes involved are integrated in such a way that they bear this causal relationship to one another and, thus, together figure into some causal generalization. This is why it is apt to think of such causal relationships as modular parts of the causal processes in which they occur.

Any complex causal process includes potentially many modular parts. This is because many distinct causal relationships are involved in the causal process, relationships that figure into distinct causal generalizations. For this reason, the context of inquiry is crucial to establishing the nature of the best explanation of an event that results from a complex causal process. The research interests that establish the context of inquiry focus attention on a particular causal relationship. This determines the modular part of the causal process that best explains the event in question, in the context at hand.

3. Explaining Phenotypic Traits. My argument that the optimality approach has a permanent role to play in evolutionary study proceeds from the account of best explanations developed in the previous section. Some evolutionary phenomena are, in certain important contexts of inquiry, best explained by optimality models. The phenomena that optimality and game-theoretic models can best explain are the phenotypic outcomes of long-term evolution by natural selection, provided such a model is applicable. Optimality explanations qualify as the best explanations of these sorts of events only in contexts of inquiry that favor the inclusion of purely phenotypic information. Such contexts arise when researchers seek to understand the organism-environment interactions and resulting selection pressures that led to the observed phenotype(s).

In certain circumstances, optimality models meet the conditions for best explanations that I set out above. The first condition is that the explanation must represent the causes that figure into the causal relationship of interest in the relevant context of inquiry. The contexts of inquiry in which optimality models best explain phenotypic evolutionary outcomes are those in which the ecological influences on the course of selection are of particular interest. Such contexts focus attention on the causal relationship obtaining between $(a)$ natural selection acting on a certain phenotypic trait, and (b) the value(s) of this trait present in the population. It is, of course, this very causal relationship that optimality models represent. The fitness function-and, for game-theoretic models, the initial distribution of trait values - is used to predict what trait value(s) will prevail in the population, given the range of possible trait values and any constraints.

The sort of causal relationship represented by optimality models figures into a particular kind of causal generalization. Fitness-conferring inter- 
actions between organism and environment have predictable consequences for the evolution of a population, in certain conditions. Eshel et al. (1998) and Eshel and Feldman (2001) demonstrate that, provided that the selection regime acting on a phenotypic trait remains constant, "the theory of long-term evolution predicts convergence to either an optimum or an ESS (evolutionarily stable strategy) regardless of the genetic system" (Eshel and Feldman 2001, 186). So long as the selection pressure is constant for a period of time sufficient for the introduction of a large number of mutations, the predictions of an optimality model can be expected to obtain. This is not so in instances of short-term evolution or when there is environmental change which affects fitness values. In these latter cases, optimality and game-theoretic models will not predictably apply.

This work supports the idea that the causal relationship picked out by an optimality model figures into a certain kind of causal generalizationthat is, the relationship is predictable, given the satisfaction of certain assumptions. The information supplied by an optimality model thus qualifies as a modular part of the evolutionary process, in the sense established above. For the long-term evolutionary change of a population in a constant environment, one can predict the phenotypic trait value(s) in the population based on information regarding how natural selection acts on the trait in question. I suggested at the beginning of this section that instances of this sort of evolutionary change are the proper explananda for optimality models. Optimality models represent a causal relationship of particular interest in the given context of inquiry, and this causal relationship figures into a causal generalization applicable to other populations, populations that might differ in the features that optimality models neglect.

The second condition outlined above is that a fully satisfactory explanation should meet the criteria of explanatory adequacy. Optimality and game-theoretic models are typically deterministic models. Thus, when an optimality model issues an accurate prediction regarding the event to be explained, then it will satisfy the first criterion of explanatory adequacy. The second criterion is a bit trickier. Sometimes, even an optimality model that correctly represents the selection dynamics and issues an accurate prediction may fail to satisfy this criterion. Yet there are plenty of instances in which the second criterion is met. Indeed, the customary assumptions of optimality models succeed for the most part for the same reason that this condition is met. For instance, the assumption that a particular set of phenotypes is available typically succeeds when any unrepresented constraints do not significantly affect the expected outcome.

Finally, optimality models also meet the third condition outlined above. That is, optimality models are maximally general explanations of the designated evolutionary phenomena, in the designated contexts of inquiry, 
within the limits created by meeting the first two conditions. It is this feature of optimality explanations that differentiates them from competing explanations that also incorporate genetic information. Such models are less general than optimality models and thus do not meet this third condition for the explananda and the contexts of inquiry that are my present focus.

When the focus is on selection as a cause of phenotypic evolutionary change, certain phenotypic outcomes of long-term evolution by natural selection are best explained by optimality models. An optimality model focuses on a particular modular part of the causal process leading to the observed phenotype. In so doing, it shows how the phenomenon to be explained fits into a pattern of phenotypic evolutionary change. The genetic causes of a single phenotype may vary, so too might the developmental pathways leading from genotype to phenotype. These are variations in the causal process by which the phenomenon is brought about. Yet, regardless of such variability, certain effects can be expected on the basis of the applicable optimality model (if one in fact is applicable).

As Brown says of game-theoretic models in particular,

Evolutionary game theory offers an exciting perspective on evolution by natural selection and its consequences. It differs from and complements genetical approaches to evolution by natural selection, in that game theory places no special value on genetic constraints relative to the other biophysical constraints that determine the size, dimension, and character of the strategy set. (Brown 2001, 154)

This holds of optimality models in general. Such models capture how natural selection works on the available variation in the long-term to produce certain phenotypic results. The instances of evolution to which a particular optimality model applies have these results in common, despite potential differences in, for example, details of genetic transmission, population size, etc. When the action of selection on phenotypes is of primary interest, optimality models best capture the relevant causal generalizations.

Incorporating genetic information into models does not add to these sorts of explanations, but would actually detract from them. An optimality model highlights a modular part of the causal process that grounds certain phenotypic generalizations. Incorporating information about other parts of the causal process can only obfuscate the relationship between the instance to be explained and other, relevantly similar instances. For this reason, I disagree with Lewontin's (1979) assessment that the optimality approach should be replaced by models explicitly incorporating genetic dynamics whenever possible. Optimality models will, I submit, always have an explanatory role to which they are best suited. 
This is, of course, not to say that optimality models are always the best explanations for evolutionary change. In different contexts of inquiry or with different explananda, other kinds of information become explanatorily relevant, for example, genetic or developmental information. Relatedly, my point should not be confused with the idea that phenotypic models are more general than genetic or synthetic genetic-phenotypic models, and thus better explanations. The context-sensitivity of explanation is an important feature of my view. Genetic models offer their own sort of generality, and I have no doubt they best explain certain phenomena, in certain contexts of inquiry. Models that bring together genetic and ecological information probably also best explain certain events in some contexts of inquiry. Each of these approaches - the purely phenotypic, the purely genetic, and the synthesis of the two-facilitates its own sort of evolutionarily valuable causal generalizations.

A good example of an optimality explanation is provided by work done on the latitudinal variation in the clutch size of birds (Ashmole 1963). According to The Birder's Handbook (Ehrlich et al. 1988),

Ornithologist N. P. Ashmole has offered an explanation of [. . .] the increase in the number of eggs per set from equator to pole. Such "latitudinal variation" in clutch size is related to the amount of food produced per unit area of habitat. More specifically, clutch size is positively related to resource abundance during the breeding season relative to the density of bird populations (abundance per unit area) at that time. If, when the birds are not breeding, their population sizes are limited by food shortages, then population density would be low at egg-laying time. And if resources increase only slightly during the breeding season, then natural selection would not favor large clutches, since food for the hatchlings would be limited. But if the increase in food were large during the breeding season, then, everything else being equal, raising a large brood should be possible. Thus the largest clutches should be found in high latitudes, where there is an enormous increase in productivity in the spring and summer (as anyone who has braved northern mosquitoes knows only too well), and the smallest clutch sizes might be expected in nonseasonal tropical rain forests, where productivity is rather uniform throughout the year.

In order to explain the latitudinal variation in clutch size observed in many species of birds, this passage cites information about why this latitudinal difference is advantageous to birds. In high latitudes, there is a great difference between the resources available in winter and in summer. This has two results: bird populations dwindle during the winter, and there are prolific sources of food for birds in the summer. This means 
that hatchlings have access to many resources, and there is a relatively small amount of competition for these resources. It is thus in birds' best evolutionary interest to have large clutches, for it is likely that the environment will be able to support many hatchlings.

The background for this explanation is the idea that natural selection favors whatever clutch size will produce the most surviving young, regardless of other features which may vary between individuals, populations, and species of birds. This is, then, an optimality explanation for this feature of average clutch size in birds. ${ }^{4}$ The fact that natural selection favors a higher number of offspring when the fluctuation between resource abundance and shortage is largest leads to the observed latitudinal cline in clutch size.

The causal relationship between selection pressures from fluctuation in resource abundance and the resultant average clutch size is captured by an optimality model. In any particular instance of a phenotype becoming common in a population of birds according to this pattern, much of the causal process does not appear in the optimality explanation. Perhaps genotypes causally related to certain clutch sizes are selectively advantaged. Perhaps a sort of phenotypic plasticity is instead selected for. Developmental pathways of one sort or another are involved. In various other contexts, these pieces of the causal process will each deserve their own focus. But the optimality explanation focuses on one modular part of the complex causal process, the modular part that figures into a causal generalization about fluctuation in resource abundance and clutch size. This generalization is made most perspicuous and is recognized as most widely applicable when the explanation is kept as general as possible. The explanation thus rightly neglects other information, for example, about genetics and development.

In my view, then, the optimality approach has a long-term role assured in evolutionary study. When plenty of data and computing power are available, incorporating genetic information may very well lead to better predictions of evolutionary change. But this does not threaten the explanatory role of the optimality approach. When explaining long-term phenotypic evolution by natural selection with a particular interest in the fitness effects of organism-environment interactions, optimality explanation is often a successful approach. Certain questions are simply best answered by optimality models.

4. As such, it is applicable only when the assumptions of an optimality model are satisfied. The passage quoted here errs on the side of making the assumption of optimality appear universal. This is problematic, given the well-established concerns with strong forms of adaptationism. 


\section{REFERENCES}

Ashmole, N. P. (1963), "The Regulation of Numbers of Tropical Oceanic Birds", Ibis 103b: 458-473.

Brown, Joel S. (2001), "Fit of Form and Function, Diversity of Life, and Procession of Life as an Evolutionary Game", in Steven Hecht Orzack and Elliott Sober (eds.), Adaptationism and Optimality. Cambridge: Cambridge University Press, 114-160.

Ehrlich, Paul, David Dobkin, and Darryl Wheye (1988), The Birder's Handbook. New York: Simon \& Schuster.

Eshel, Ilan, and Marcus W. Feldman (2001), "Optimality and Evolutionary Stability under Short-Term and Long-Term Selection", in Steven Hecht Orzack and Elliott Sober (eds.), Adaptationism and Optimality. Cambridge: Cambridge University Press, 114-160.

Eshel, Ilan, Marcus W. Feldman, and Aviv Bergman (1998), "Long-Term Evolution, ShortTerm Evolution, and Population Genetic Theory", Journal of Theoretical Biology 191: 391-396.

Garfinkel, Alan (1981), Forms of Explanation: Rethinking the Questions in Social Theory. New Haven, CT: Yale University Press.

Jackson, Frank, and Philip Pettit (1992), "In Defense of Explanatory Ecumenism", Economics and Philosophy 8: 1-21.

Lewontin, Richard (1979), "Fitness, Survival, and Optimality", in David Horn, Gordon Stairs, and Rodger Mitchell (eds.), Analysis of Ecological Systems, Third Annual Biosciences Colloquium. Columbus: Ohio State University Press, 3-21.

Mayr, Ernst (1961). "Cause and Effect in Biology", Science 134: 1501-1506.

Putnam, Hilary (1975), "Philosophy and Our Mental Life", in Philosophical Papers, vol. 2. London: Cambridge University Press, 291-303.

Sober, Elliott (1999), "The Multiple Realizability Argument against Reduction", Philosophy of Science 66: 542-564.

(2003), "Two Uses of Unification", in Friedrich Stadler (ed.), The Vienna Circle and Logical Empiricism: Re-evaluation and Future Perspectives. Dordrecht: Kluwer, 205216.

Strevens, Michael (2004), "The Causal and Unification Accounts of Explanation UnifiedCausally", Noûs 38: 154-179. 